\title{
Analytical Formulation for Electromagnetic Leakage Field to Transmission Line Coupling through Covered Apertures of Multiple Enclosures
}

\author{
Jianhong Hao, Yanfei Gong, Luhang Jiang, and Jieqing Fan \\ School of Electrical and Electronic Engineering, North China Electric Power University, Beijing, China \\ Correspondence should be addressed to Yanfei Gong; yuxin880323@126.com
}

Received 31 October 2016; Revised 26 January 2017; Accepted 7 February 2017; Published 1 March 2017

Academic Editor: Anna Richelli

Copyright (C) 2017 Jianhong Hao et al. This is an open access article distributed under the Creative Commons Attribution License, which permits unrestricted use, distribution, and reproduction in any medium, provided the original work is properly cited.

\begin{abstract}
An efficient analytical model has been developed for predicting the electromagnetic leakage field coupling with a lossless twoconductor transmission line (TL) through covered apertures of multiple enclosures. The analytical results have been successfully compared with those from the full-wave simulation software CST over a broad frequency range. The analytical model can be employed to analyze the effect of different factors including the position and the direction of the electric dipole, the conductivity of the conductive sheet, the quantity of the aperture, and the direction of the TL on the induced currents. Besides, it can also deal with apertures in multiple sides of the enclosures.
\end{abstract}

\section{Introduction}

Due to the increasing complexity and density of high speed integrated circuits and electronic devices, the problems of electromagnetic interference (EMI) have become considerably common and severe. Electromagnetic shielding, one of the primary technical measures for suppressing the EMI via field coupling channel, has been widely used in the electromagnetic compatibility (EMC) design. It can be simply implemented by encasing vulnerable circuits and components within a metallic box to protect them from the EMI. Generally, a shielding enclosure is divided into multiple spaces where massive circuits and components are distributed for the purpose of reducing the mutual EMI among them. However, significant electromagnetic leakage can occur from the adjacent space of the enclosure through slots, apertures, connecting lines, and so forth, in which case the transient voltage and current induced by the EMI may degrade the performance of some sensitive components of the equipment and even damage them. The electromagnetic field coupling with wires, PCBs, and other objects located in an enclosure through apertures has become an important issue in recent years [1-3]. In particular, the electromagnetic field coupling with wires, an indispensable part of electronic systems used for circuital connection and signal transmission, has attracted much more attention in circuit related EMI problems [4-7].

In order to evaluate the response of the wires enclosed within an enclosure to the electromagnetic field, previous studies were carried out by numerical methods [4, 5]. Although such methods are robust and accurate, every detail of the wires has to be analyzed, thus requiring large computational resources. As a result, analytical approaches [6-11], which provide a much faster and more convenient technique of calculation and analysis of the effect of different parameters on the voltage and current induced on the wires, have been especially used in predicting the electromagnetic field coupling with wires. In the previous studies, $\mathrm{Li}$ et al. [6] have implemented the electromagnetic topology theory for electromagnetic field coupling with a two-conductor transmission line (TL) in a rectangular enclosure with apertures illuminated by an external plane wave. Based on the equivalent lumped-pi circuit model, the electromagnetic field coupling with a single-conductor TL in a rectangular enclosure excited by an internal electric dipole is investigated in [7]. Konefal et al. [8,9] have applied the intermediate 
level circuit model (ILCM) for the field coupling through apertures or covered apertures irritated by an external plane wave. The ILCM technique has also been used to study the electromagnetic coupling between lines and loops inside a rectangular enclosure $[10,11]$. References [6-11] concentrate on the field problems of a single enclosure. However, there are few studies for the electromagnetic field coupling with TLs in multiple apertured enclosures. Indeed, with the rapid development of large-scale integrated circuits, the phenomena of electromagnetic leakage from an enclosure into another through apertures are more common. Unlike [7] in which the excitation source and the TL are placed in the same enclosure, they are required to be distributed into different enclosures when the electromagnetic leakage field is taken into consideration. In addition, due to the rapid development of material science and technology, an increasing number of conductive materials with good flexibility and low weight have been used in the design of shielding enclosures $[12,13]$. Sometimes, they are employed to close apertures to improve the shielding effectiveness of an enclosure [9]. Similarly, they can also be used to close apertures of adjacent multiple enclosures in order to decrease the electromagnetic leakage from them.

In the paper, an efficient analytical model has been proposed for predicting the electromagnetic leakage field coupling with a lossless two-conductor TL through covered apertures of multiple enclosures. Firstly, the TL is located in a metallic rectangular enclosure, and the leakage field is excited by an electric dipole inside another adjacent metallic rectangular enclosure with an aperture covered by a conductive sheet. A significant part of this work focuses on deriving the leakage field into the enclosure containing the TL through the covered aperture by using the dyadic Green function and the boundary condition of the electromagnetic wave normally incident upon an infinite conducting surface. Then, the electric current induced at the termination of the TL is calculated by the combination of the equivalent lumpedT circuit model of the TL and the Agrawal coupling model. The analytical model can also be extended to deal with the case that the TL is located outside the multiple enclosures. Finally, the analytical results are compared with those from the full-wave simulation software CST, which shows that the present analytical model is useful to achieve an efficient and relatively accurate prediction of the electromagnetic leakage field to TL coupling through covered apertures over a broad frequency range. Thus, the analytical model then is employed to analyze the effect of different factors including the position and the direction of the electric dipole, the conductivity of the conductive sheet, the quantity and the position of the aperture, and the direction of the TL on the induced current. However, it should be noted that the reradiated field created by the TL has been ignored in the proposed model.

\section{The Establishment of Analytical Model}

2.1. TL in the Enclosures. The geometry of the analytical model is shown in Figure 1(a). It consists of two adjacent metallic rectangular enclosures connected by a metallic plate with a circular aperture (the radius of the circular aperture is $r_{a}$ ) covered by a conductive sheet of thickness $t$ and conductivity $\sigma$, and these enclosures are considered as perfect electric conductors (PEC). Figure 1(b) shows the geometry of the metallic plate with the covered circular aperture. The center point of the aperture is located at $\left(x_{0}, y_{0}, z_{0}\right)$. The $x$, $y$, and $z$ dimensions of both enclosures (enclosure 1 and enclosure 2) are $x_{e}, y_{e}$, and $z_{e}$, respectively. The interference source is an electric dipole oriented along the $y$-axis and located at $\left(x_{s}, y_{s}, z_{s}\right)$ in enclosure 1 . Enclosure 2 contains a parallel lossless two-conductor TL oriented along the $x$-axis of both length $L$ and radius $r_{t}$ and height $h$ (the distance between the center of the TL and enclosure 2). The distance between the two wires is $d_{t}$, and the distance of the TL to the metallic plate is $d . Z_{1}$ and $Z_{2}$ are the termination loads, respectively, at the left and right ends of the line. The center point of the TL and the observation point are, respectively, located at $\left(x_{t}, y_{t}, z_{t}\right)$ and $(x, y, z)$ in enclosure 2 . The corresponding side view of the TL is shown in Figure 1(c).

Generally, rectangular enclosures modes are composed of TE mode and TM mode. In our model, the TE mode cannot be inspired because there is no $y$-component of electric field, and thus the electromagnetic field inside enclosure 1 is only represented by the TM mode.

The problem of computing the electric current induced at the termination loads $Z_{1}$ and $Z_{2}$ is divided into three steps. First of all, we derive the leakage field inside the empty enclosure 2 excited by the electric dipole by using the dyadic Green function and the boundary condition of the electromagnetic wave normally incident upon an infinite conducting surface. Secondly, the TL is modeled by the equivalent $\mathrm{T}$ circuit model. Finally, the intermediate circuit model is constructed between the leakage field and the TL elements based on the Agrawal coupling model. These steps will be described in detail as follows.

2.1.1. Electromagnetic Leakage Field Formulation. In order to derive the leakage field in enclosure 2 without the TL through the covered aperture excited by the electric dipole located in enclosure 1, we divide the problem into three subproblems: (a) the problem of obtaining the field distribution in enclosure 1 with the aperture short-circuited; (b) the problem of determining the tangential electric field on the right surface of the conductive sheet; (c) the problem of calculating the leakage field based on the equivalent magnetic current on the right surface of the conductive sheets.

According to [14] and by using Faraday's electromagnetic induction law, the component of magnetic field $H_{x}^{1}$ inside enclosure 1 with the aperture short-circuited produced by the $y$-directed electric dipole can be obtained:

$$
\begin{aligned}
& H_{x}^{1}(x, y, z)=\frac{-I d l}{x_{e} y_{e} z_{e}} \sum_{m=0}^{\infty} \sum_{l=0}^{\infty} \Gamma_{m l}\left(\frac{y_{e}}{2 k_{1}}\right) \sin ^{-1}\left(k_{1} y_{e}\right) \\
& \cdot\left\{\cos k_{1}\left(y+y_{s}-y_{e}\right)+\cos k_{1}\left(\left|y-y_{s}\right|-y_{e}\right)\right\}, \\
& H_{y}^{1}(x, y, z)=0,
\end{aligned}
$$

where $I$ and $d l$ are the current and length of the electric dipole, respectively; $m$ and $l$ are the mode index numbers 


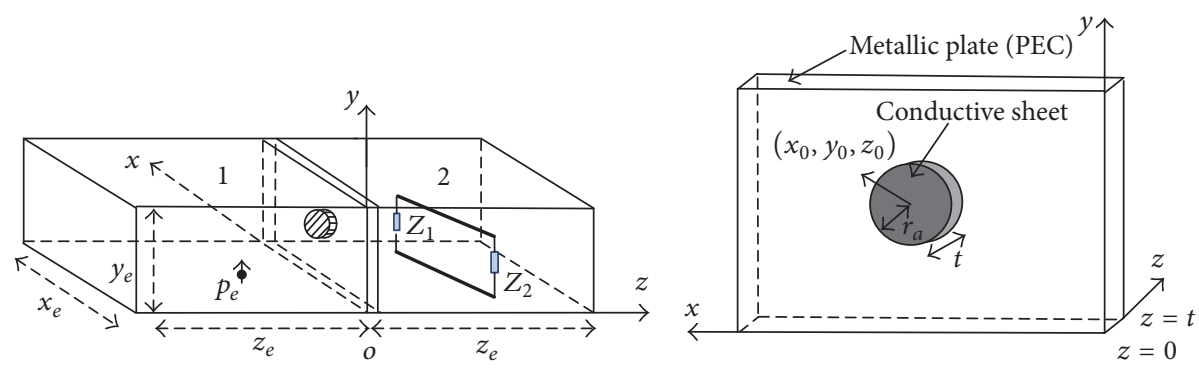

(a)

(b)

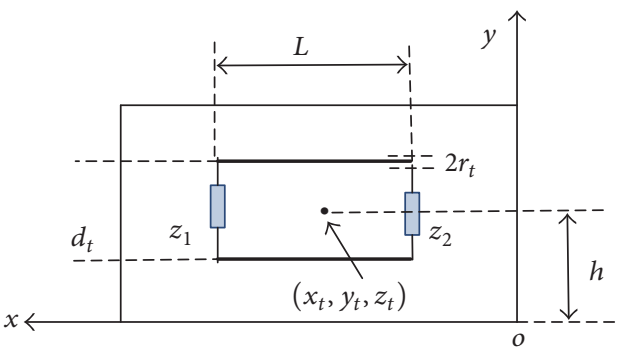

(c)

Figure 1: (a) Two adjacent metallic rectangular enclosures connected by a metallic plate with an aperture covered by a conductive sheet excited by an electric dipole in enclosure 1 and containing a two-conductor TL in enclosure 2. (b) The geometry of the metallic plate with the covered circular aperture. (c) The side view of the TL corresponding to (a).

of enclosure 1 along the $x$-axis and the $z$-axis, respectively, and

$$
\begin{aligned}
k_{1} & =\sqrt{k_{0}^{2}-\left(\frac{m \pi}{x_{e}}\right)^{2}-\left(\frac{l \pi}{z_{e}}\right)^{2}}, \\
\Gamma_{m l} & =\varepsilon_{0 m} \varepsilon_{0 l}\left(\frac{l \pi}{z_{e}}\right) \cos \left(\frac{l \pi z}{z_{e}}\right) \sin \left(\frac{l \pi z_{s}}{z_{e}}\right) \sin \left(\frac{m \pi x}{x_{e}}\right) \\
\cdot & \sin \left(\frac{m \pi x_{s}}{x_{e}}\right),
\end{aligned}
$$

where $k_{0}=\omega \sqrt{\mu_{0} \varepsilon_{0}}(1-j /(2 Q))$, and $Q\left(Q=Q_{0}\left(f_{0}\right)\right.$. $\left.\left(f / f_{0}\right), Q_{0}\left(f_{0}=0.1 \mathrm{GHz}\right)=1000\right)$ is the quality factor which represents the losses of the enclosure; $\omega$ is the angular frequency; $\varepsilon_{0}$ is the permittivity in vacuum; $\mu_{0}$ is the vacuum permeability; $\varepsilon_{0 m}\left(\varepsilon_{0 l}\right)=1$ for $m(l)=0$ and $\varepsilon_{0 m}\left(\varepsilon_{0 l}\right)=2$ for $m(l) \neq 0$.

By enforcing the boundary condition of the electromagnetic wave normally incident upon an infinite conducting surface at $z=t$

$$
\begin{gathered}
\left.E_{i y}\right|_{z=t}+\left.E_{r y}\right|_{z=t}=\left.E_{t y}\right|_{z=t}, \\
\left.H_{i x}\right|_{z=t}+\left.H_{r x}\right|_{z=t}=\left.H_{t x}\right|_{z=t},
\end{gathered}
$$

where $E_{i y}$ and $H_{i x}$ are the incident electric field along the $y$-axis and the incident magnetic field along the $x$-axis in the region of conductive sheets, respectively; $E_{r y}$ and $H_{r x}$ are the reflected electric field along the $y$-axis and the reflected magnetic field along the $x$-axis in the region of conductive sheets, respectively; $E_{t y}$ and $H_{t x}$ are the transmission electric field along the $y$-axis and the transmission magnetic field along the $x$-axis in enclosure 2 , respectively.
We can construct a connection between the transmission electric field $E_{t}$ on the right surface of the conductive sheets and the incident magnetic field $H_{i}$ on the left surface of the sheets using $\mathbf{E}_{t}=\mathbf{e}_{n} \times\left[\eta(d) \mathbf{H}_{i}\right]$, where $\mathbf{e}_{n}$ is the unit normal vector of the right surface of the sheets and here is equal to $\mathbf{e}_{z}$. If $\mathbf{H}_{i}$ is approximated by the magnetic field $\mathbf{H}$ in enclosure 1 when it is totally closed, we can obtain as [15]

$$
\begin{aligned}
& E_{t y}=\frac{\left(-2 \eta_{1} \eta_{2}\right)}{\left(\eta_{1}+\eta_{2}\right) e^{j k_{2} t}-\left(\eta_{1}-\eta_{2}\right) e^{-j k_{2} t}} H_{x}^{1}=\eta(t) H_{x}^{1}, \\
& E_{t x}=\eta(t) H_{y}^{1}=0,
\end{aligned}
$$

where $\eta_{1}=\sqrt{\mu_{0} / \varepsilon_{0}}, k_{2}=\omega \sqrt{\mu_{0} \varepsilon_{2}}, \varepsilon_{2}=\varepsilon_{0}-j(\sigma / \omega), \eta_{2}=$ $\sqrt{\mu_{0} / \varepsilon_{2}}$.

Considering that the right surface of the sheet is located on the $z=t$ plane, the equivalent surface magnetic current $\mathbf{M}$ has both the $x$-and $y$-components. Using the surface equivalence principle and the image theory, the covered aperture can be replaced by the equivalent magnetic current on the right surface of the sheet as

$$
\begin{aligned}
\mathbf{M} & =2\left(\mathbf{E}_{t y}+\mathbf{E}_{t x}\right) \times \mathbf{e}_{z}=2 E_{t y} \mathbf{e}_{x}-2 E_{t x} \mathbf{e}_{y} \\
& =M_{x} \mathbf{e}_{x}+M_{y} \mathbf{e}_{y} .
\end{aligned}
$$

The electromagnetic fields in enclosure 2 in terms of the electric vector potential $\mathbf{A}_{m}$ are given as

$$
\begin{aligned}
& \mathbf{E}=-\left(\frac{1}{\varepsilon_{0}}\right) \nabla \times \mathbf{A}_{m}, \\
& \mathbf{H}=\left(\frac{-j \omega}{k_{0}^{2}}\right)\left(k_{0}^{2} \mathbf{A}_{m}+\nabla \nabla \cdot \mathbf{A}_{m}\right)
\end{aligned}
$$


$\mathbf{A}_{m}$ satisfies the inhomogeneous wave equation:

$$
\nabla^{2} \mathbf{A}_{m}+k_{0}^{2} \mathbf{A}_{m}=-\varepsilon_{0} \mathbf{M}
$$

Using $\mathbf{A}_{m}=\iint_{S^{\prime}} \overline{\overline{\mathbf{G}}}_{m}\left(\mathbf{r}, \mathbf{r}^{\prime}\right) \cdot \mathbf{M}\left(\mathbf{r}, \mathbf{r}^{\prime}\right) d s^{\prime}$, where $\overline{\overline{\mathbf{G}}}_{m}\left(\mathbf{r}, \mathbf{r}^{\prime}\right)$ is the dyadic Green function of enclosure $2, \mathbf{r}$ and $\mathbf{r}^{\prime}$ are, respectively, the observation and the equivalent magnetic current source vectors, and $S^{\prime}$ denotes the region which the aperture covers, (8) is substituted as

$$
\nabla^{2} \overline{\overline{\mathbf{G}}}_{m}\left(\mathbf{r}, \mathbf{r}^{\prime}\right)+k_{0}^{2} \overline{\overline{\mathbf{G}}}_{m}\left(\mathbf{r}, \mathbf{r}^{\prime}\right)=-\varepsilon_{0} \overline{\overline{\mathbf{I}}} \delta\left(\mathbf{r}-\mathbf{r}^{\prime}\right)
$$

where the unit dyad $\overline{\overline{\mathbf{I}}}=\mathbf{e}_{x} \mathbf{e}_{x}+\mathbf{e}_{y} \mathbf{e}_{y}$.

According to [16], the $x x$ - and $y y$-components of $\overline{\overline{\mathbf{G}}}_{m}\left(\mathbf{r}, \mathbf{r}^{\prime}\right)$ in (9) can be written as

$$
\begin{aligned}
& G_{m x x}=-\sum_{m^{\prime}=0}^{\infty} \sum_{n^{\prime}=0}^{\infty} \frac{\varepsilon_{0} \varepsilon_{0 m^{\prime}} \varepsilon_{0 n^{\prime}}}{\left(x_{e} y_{e} k_{z}\right)} \sin \left(k_{x m^{\prime}} x\right) \cos \left(k_{y n^{\prime}} y\right) \\
& \cdot \frac{\cos \left(k_{z}\left(z-z_{e}\right)\right)}{\sin \left(k_{z}\left(z_{e}-t\right)\right)} \sin \left(k_{x m^{\prime}} x^{\prime}\right) \\
& \cdot \cos \left(k_{y n^{\prime}} y^{\prime}\right) \delta\left(z^{\prime}-t\right), \\
& G_{m y y}=-\sum_{m^{\prime}=0}^{\infty} \sum_{n^{\prime}=0}^{\infty} \frac{\varepsilon_{0} \varepsilon_{0 m^{\prime}} \varepsilon_{0 n^{\prime}}}{\left(x_{e} y_{e} k_{z}\right)} \cos \left(k_{x m^{\prime}} x\right) \sin \left(k_{y n^{\prime}} y\right) \\
& \cdot \frac{\cos \left(k_{z}\left(z-z_{e}\right)\right)}{\sin \left(k_{z}\left(z_{e}-t\right)\right)} \cos \left(k_{x m^{\prime}} x^{\prime}\right) \\
& \cdot \sin \left(k_{y n^{\prime}} y^{\prime}\right) \delta\left(z^{\prime}-t\right),
\end{aligned}
$$

where $\left(m^{\prime}, n^{\prime}\right)$ are the mode index numbers of enclosure 2; $k_{x m^{\prime}}=m^{\prime} \pi / x_{e}, k_{y n^{\prime}}=n^{\prime} \pi / y_{e}$, and $k_{z}=\sqrt{k_{0}^{2}-k_{x m^{\prime}}^{2}-k_{y n^{\prime}}^{2}}$.

Substituting (5) and (10) into the expression of the electric vector potential $\mathbf{A}_{m}$, the $x$ - and $y$-components of it are obtained as

$$
\begin{aligned}
& A_{m x}=\iint_{S^{\prime}} G_{m x x} M_{x} d s^{\prime}=2 \eta(t) \\
& \cdot \sum_{m^{\prime}=0}^{\infty} \sum_{n^{\prime}=0}^{\infty} \frac{-\varepsilon_{0} \varepsilon_{0 m^{\prime}} \varepsilon_{0 n^{\prime}}}{x_{e} y_{e} k_{z} \sin \left(k_{z}\left(z_{e}-t\right)\right)} \cos \left(k_{z}\left(z-z_{e}\right)\right) \\
& \cdot \iint_{S^{\prime}} H_{x}^{1}\left(\mathbf{r}^{\prime}\right) \sin \left(k_{x m^{\prime}} x^{\prime}\right) \cos \left(k_{y n^{\prime}} y^{\prime}\right) d s^{\prime} \\
& \cdot \sin \left(k_{x m^{\prime}} x\right) \cos \left(k_{y n^{\prime}} y\right) \\
& A_{m y}=\iint_{S^{\prime}} G_{m y y} M_{y} d s^{\prime}=0 .
\end{aligned}
$$

The electromagnetic fields inside enclosure 2 are then obtained from (6), (7), and (11) as

$$
E_{x}^{2}=0,
$$

$$
\begin{aligned}
& E_{y}^{2}=-2 \eta(t) \sum_{m^{\prime}=0}^{\infty} \sum_{n^{\prime}=0}^{\infty} \frac{\left(\varepsilon_{0 m^{\prime}} \varepsilon_{0 n^{\prime}}\right) \sin \left(k_{x m^{\prime}} x\right)}{x_{e} y_{e} \sin \left(k_{z}\left(z_{e}-t\right)\right)} \\
& \cdot \cos \left(k_{y n^{\prime}} y\right) \sin \left(k_{z}\left(z-z_{e}\right)\right) \\
& \iint_{S^{\prime}} H_{x}^{1}\left(x^{\prime}, y^{\prime}, z^{\prime}\right) \sin \left(k_{x m^{\prime}} x^{\prime}\right) \cos \left(k_{y n^{\prime}} y\right) d s^{\prime}, \\
& E_{z}^{2}=-2 \eta(t) \sum_{m^{\prime}=0}^{\infty} \sum_{n^{\prime}=0}^{\infty} \frac{\varepsilon_{0 m^{\prime}} \varepsilon_{0 n^{\prime}} k_{y n^{\prime}} \sin \left(k_{x m^{\prime}} x\right)}{x_{e} y_{e} k_{z} \sin \left(k_{z}\left(z_{e}-t\right)\right)} \\
& \cdot \sin \left(k_{y n^{\prime}} y\right) \cos \left(k_{z}\left(z-z_{e}\right)\right) \\
& \text { - } \iint_{S^{\prime}} H_{x}^{1}\left(x^{\prime}, y^{\prime}, z^{\prime}\right) \sin \left(k_{x m^{\prime}} x^{\prime}\right) \cos \left(k_{y n^{\prime}} y\right) d s^{\prime}, \\
& H_{x}^{2}=2 \eta(t) \frac{j \omega}{k_{0}^{2}} \sum_{m^{\prime}=0}^{\infty} \sum_{n^{\prime}=0}^{\infty} \frac{\varepsilon_{0} \varepsilon_{0 m^{\prime}} \varepsilon_{0 n^{\prime}}\left(k_{0}^{2}-k_{x m^{\prime}}^{2}\right)}{x_{e} y_{e} k_{z} \sin \left(k_{z}\left(z_{e}-t\right)\right)} \\
& \cdot \sin \left(k_{x m^{\prime}} x\right) \cos \left(k_{y n^{\prime}} y\right) \\
& \text {. } \iint_{S^{\prime}} H_{x}^{1}\left(x^{\prime}, y^{\prime}, z^{\prime}\right) \sin \left(k_{x m^{\prime}} x^{\prime}\right) \cos \left(k_{y n^{\prime}} y\right) d s^{\prime} \\
& \cdot \cos \left(k_{z}\left(z-z_{e}\right)\right) \text {, } \\
& H_{y}^{2}=-2 \eta(t) \frac{j \omega}{k_{0}^{2}} \sum_{m^{\prime}=0}^{\infty} \sum_{n^{\prime}=0}^{\infty} \frac{\varepsilon_{0} \varepsilon_{0 m^{\prime}} \varepsilon_{0 n^{\prime}} k_{x m^{\prime}} k_{y n^{\prime}}}{x_{e} y_{e} k_{z} \sin \left(k_{z}\left(z_{e}-t\right)\right)} \\
& \cdot \cos \left(k_{x m^{\prime}} x\right) \sin \left(k_{y n^{\prime}} y\right) \\
& \text {. } \iint_{S^{\prime}} H_{x}^{1}\left(x^{\prime}, y^{\prime}, z^{\prime}\right) \sin \left(k_{x m^{\prime}} x^{\prime}\right) \cos \left(k_{y n^{\prime}} y\right) d s^{\prime} \\
& \cdot \cos \left(k_{z}\left(z-z_{e}\right)\right) \text {, } \\
& H_{z}^{2}=-2 \eta(t) \frac{j \omega}{k_{0}^{2}} \sum_{m^{\prime}=0}^{\infty} \sum_{n^{\prime}=0}^{\infty} \frac{\varepsilon_{0} \varepsilon_{0 m^{\prime}} \varepsilon_{0 n^{\prime}} k_{x m^{\prime}}}{x_{e} y_{e} \sin \left(k_{z}\left(z_{e}-t\right)\right)} \\
& \cdot \cos \left(k_{x m^{\prime}} x\right) \cos \left(k_{y n^{\prime}} y\right) \\
& \text {. } \iint_{S^{\prime}} H_{x}^{1}\left(x^{\prime}, y^{\prime}, z^{\prime}\right) \sin \left(k_{x m^{\prime}} x^{\prime}\right) \cos \left(k_{y n^{\prime}} y\right) d s^{\prime} \\
& \cdot \sin \left(k_{z}\left(z-z_{e}\right)\right) \text {. }
\end{aligned}
$$

2.1.2. Equivalent T Circuit Model of TL. In order to model the electric leakage field coupling with the lossless twoconductor TL, the TL is divided into a series of elementary segments consisting of inductors and capacitors based on the equivalent lumped-T circuit model [17]. Here we have the following four assumptions.

(1) The length of the elementary unit of the TL is smaller than $\lambda / 10$, where $\lambda$ is the wavelength corresponding 


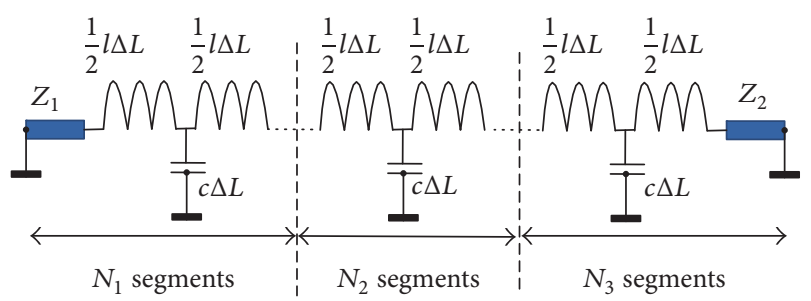

FIGURE 2: The equivalent lumped-T circuit model of TL.

to the frequency range of interest $(0.1 \sim 2.5 \mathrm{GHz}$ in our model).

(2) The distance between the two wires is much smaller than the wavelength $\left(d_{t} \ll \lambda\right)$.

(3) The radius of the TL is much smaller compared to the distance between the two wires $\left(r_{t} \ll d_{t}\right)$.

(4) The surroundings of the TL are lossless dielectric.

Figure 2 shows the equivalent $\mathrm{T}$ circuit model of the TL in which it is divided into $N$ segments $\left(N=N_{1}+\right.$ $N_{2}+N_{3}$, where $N_{1}$ and $N_{2}$ are, respectively, the segments numbers of the left and the right termination load of the TL; $N_{3}$ is the segments number of the middle part of the TL). According to assumptions (3) and (4) above, the inductance and capacitance in a line of unit-length $\Delta L$ are

$$
\begin{aligned}
& l=\frac{\mu_{0}}{\pi} \ln \frac{d_{t}}{r_{t}}, \\
& c=\frac{\pi \varepsilon_{0}}{\ln \left(d_{t} / r_{t}\right)} .
\end{aligned}
$$

Then the Agrawal coupling model is used to describe the effects of the electric leakage field on the TL.

2.2. TL Outside the Enclosures. Based on Figure 1, Figure 3 shows both of the metallic plate and the right wall of enclosure 2 have an aperture (aperture 1 of radius $r_{a}$ and aperture 2 of radius $r_{b}$ ) covered by a conductive sheet (sheet 1 of thickness $t_{1}$, conductivity $\sigma_{1}$ and sheet 2 of thickness $t_{2}$, conductivity $\sigma_{2}$ ), and the two-conductor TL is located outside the multiple enclosures. The center point of the TL and the observation point are, respectively, located at $\left(x_{t 1}, y_{t 1}, z_{t 1}\right)$ and $\left(x_{1}, y_{1}, z_{1}\right)$ outside the multiple enclosures.

In order to predict the leakage field coupling with the TL through the two covered apertures, the leakage fields outside enclosure 2 should be obtained. According to (6), we should first calculate the electric vector potential $\mathbf{A}_{m 1}$ :

$$
\mathbf{A}_{m 1}=\frac{\varepsilon_{0}}{4 \pi} \iint_{S^{\prime \prime}} \frac{\mathbf{M}_{1}\left(\mathbf{r}^{\prime \prime}\right) e^{-j k_{0} r}}{r} d s^{\prime \prime},
$$

where $S^{\prime \prime}$ denotes the area which sheet 2 covers, $r=\left|\mathbf{r}-\mathbf{r}^{\prime \prime}\right|$, and $\mathbf{M}_{1}\left(\mathbf{r}^{\prime \prime}\right)$ is the equivalent magnetic current of the right surface of the conductive sheet 2 , which has both the $x$ - and $y$-components.

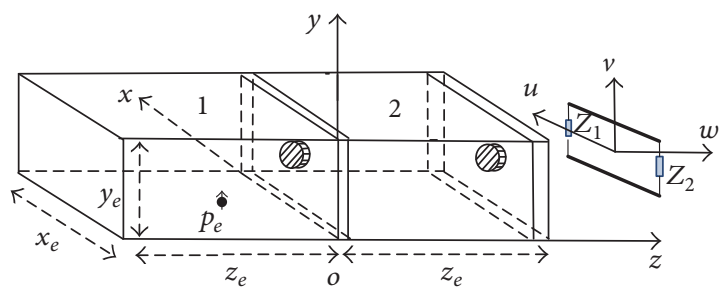

FIgURE 3: Two adjacent metallic rectangular enclosures (enclosure 1 and enclosure 2 with a covered aperture 2 at the right wall) connected by a metallic plate with a covered aperture 1 excited by an electric dipole in enclosure 1 and with a two-conductor TL outside enclosure 2 .

According to (4) and (5), the $x$-component and the $y$ component of $\mathbf{M}_{1}\left(\mathbf{r}^{\prime \prime}\right)$ are, respectively, obtained as

$$
\begin{aligned}
& M_{1 x}=2 E_{1 t y}=2 H_{x}^{2} \eta\left(t_{2}\right), \\
& M_{1 y}=-2 E_{1 t x}=-2 H_{y}^{2} \eta\left(t_{2}\right) .
\end{aligned}
$$

Therefore, we can obtain the leakage fields outside enclosure 2 by the light of (6) as

$$
\begin{aligned}
& E_{x}^{\text {out }}=\frac{-1}{4 \pi} \iint_{S^{\prime \prime}} \frac{e^{-j k_{0} r}\left(1+j k_{0} r\right)}{r^{3}}\left(z-z^{\prime \prime}\right) \\
& \cdot M_{1 y}\left(\mathbf{r}^{\prime \prime}\right) d s^{\prime \prime}, \\
& E_{y}^{\text {out }}=\frac{1}{4 \pi} \iint_{S^{\prime \prime}} \frac{e^{-j k_{0} r}\left(1+j k_{0} r\right)}{r^{3}}\left(z-z^{\prime \prime}\right) \\
& \cdot M_{1 x}\left(\mathbf{r}^{\prime \prime}\right) d s^{\prime \prime}, \\
& E_{z}^{\text {out }}=\frac{1}{4 \pi} \iint_{S^{\prime \prime}} \frac{e^{-j k_{0} r}\left(1+j k_{0} r\right)}{r^{3}}\left[\left(x-x^{\prime \prime}\right) M_{1 y}\left(\mathbf{r}^{\prime \prime}\right)\right. \\
& \left.-\left(y-y^{\prime \prime}\right) M_{1 x}\left(\mathbf{r}^{\prime \prime}\right)\right] d s^{\prime \prime} .
\end{aligned}
$$

Using the equivalent T circuit model of TL in Section 2.1 similarly, we can finally obtain the electric currents induced at the termination of the TL.

\section{Results and Discussion}

3.1. TL in the Enclosures. First, we consider a covered circular aperture of radius $r_{a}=20 \mathrm{~mm}$, thickness $t=1 \mathrm{~mm}$, and conductivity $\sigma=100 \mathrm{~S} / \mathrm{m}$. It is assumed that the dimensions of enclosure 1 and enclosure 2 are both $x_{e}=z_{e}=300 \mathrm{~mm}$ and $y_{e}=120 \mathrm{~mm}$; the TL length, radius, and height are $L=$ $100 \mathrm{~mm}, r_{t}=1 \mathrm{~mm}$, and $h=50 \mathrm{~mm}$, respectively. The distance between the two wires is $d_{t}=20 \mathrm{~mm}$, and the distance of the TL to the metallic plate is $d=150 \mathrm{~mm}$. The center point of the aperture is located at $(150,60,0.5) \mathrm{mm}$. Specially, the aperture can be located at other parts of the metallic plate, but it should not be very close to the edge of it. The electric dipole, the center point of the TL, and the observation point are located at $(152.5,20,-147.5) \mathrm{mm},(150,60,150) \mathrm{mm}$, and $(150,60,150) \mathrm{mm}$, respectively. The termination loads are 


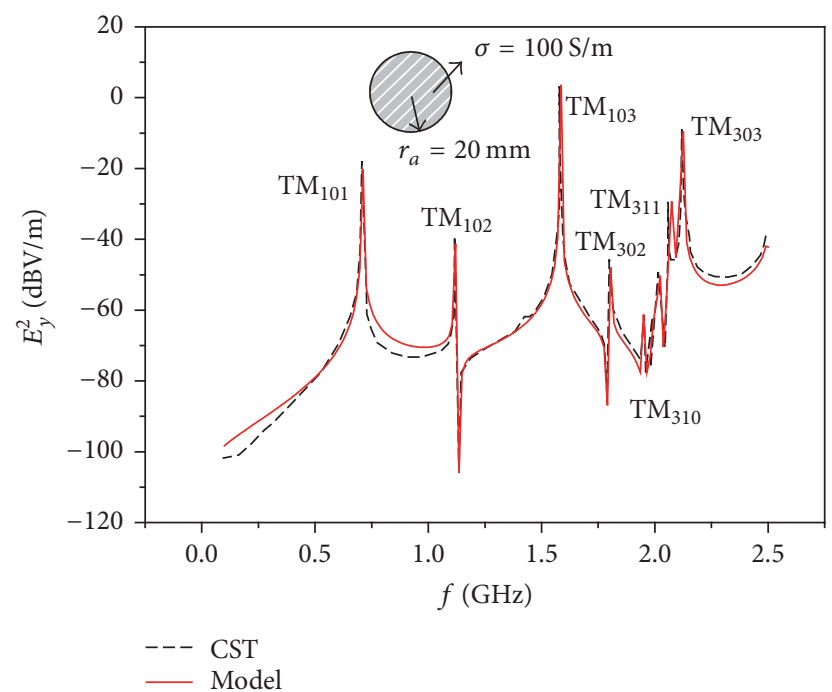

FIGURE 4: Component of electric field $E_{y}^{2}$ at $(150,60,150) \mathrm{mm}$ for a covered circular aperture from the analytical model and the CST.

$Z_{1}=Z_{2}=50 \Omega$. Here, the two-conductor TL is divided into ten segments in the equivalent circuit model, and that is enough for getting the accurate results.

In order to calculate the $y$-component of the electric field $E_{y}^{2}$ which is dominant in the three electric field components without the TL, (13) is used. Figure 4 shows the comparison of $E_{y}^{2}$ using the analytical model and the results from the CST. It can be seen that the two curves are in good agreement within a broad frequency range in Figure 4 . In our results all resonance modes have been identified corresponding to the enclosure resonant frequencies, including the $\mathrm{TM}_{101}$ resonance at $0.71 \mathrm{GHz}$, the $\mathrm{TM}_{102}$ resonance at $1.12 \mathrm{GHz}$, the $\mathrm{TM}_{103}$ resonance at $1.58 \mathrm{GHz}$, the $\mathrm{TM}_{302}$ resonance at $1.80 \mathrm{GHz}$, the $\mathrm{TM}_{310}$ resonance at $1.95 \mathrm{GHz}$, the $\mathrm{TM}_{311}$ resonance at $2.02 \mathrm{GHz}$, and the $\mathrm{TM}_{303}$ resonance at $2.12 \mathrm{GHz}$.

Figures 5 and 6 show the comparison of the electric current induced at the termination loads $Z_{1}$ and $Z_{2}$, respectively, using the analytical model and the results from the CST. It can be seen that there is a good agreement between the two curves. In the frequency range $0.1 \sim 2.5 \mathrm{GHz}$, all resonance modes $\left(\mathrm{TM}_{101}, \mathrm{TM}_{102}, \mathrm{TM}_{103}, \mathrm{TM}_{302}\right.$, and $\left.\mathrm{TM}_{303}\right)$ coupled with the TL have been identified and correspond to the enclosure resonant frequencies which have been calculated in Figure 4 . The induced current reaches its maximum values for these frequencies, indicating that the coupling between the leakage field and the TL is very strong.

Then various configurations are studied, including different positions of the electric dipole, different conductivities of the conductive sheet, different quantities of the aperture, and different directions of the TL and the electric dipole, respectively.

Figure 7 shows the electric current induced at the termination load $Z_{1}$ for different electric dipole positions of $(152.5,20,-140) \mathrm{mm},(152.5,20,-160) \mathrm{mm}$, and $(152.5,20$, $-170) \mathrm{mm}$. It can be seen that with the variation of the $z$ coordinate of the electric dipole point, the induced current

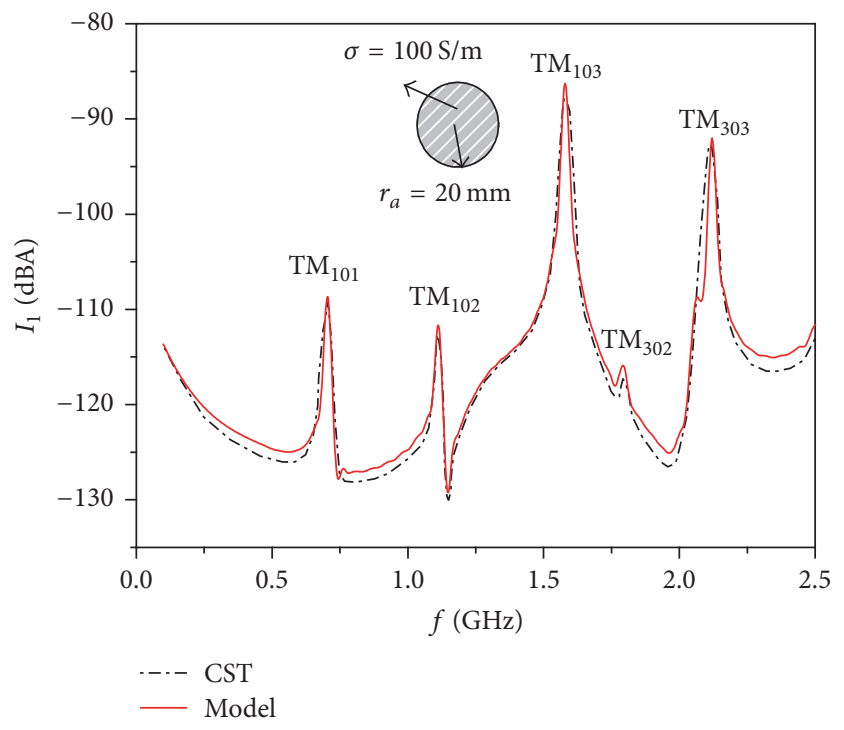

FIGURE 5: Electric current induced at termination load $Z_{1}$ for a covered circular aperture from the analytical model and the CST.

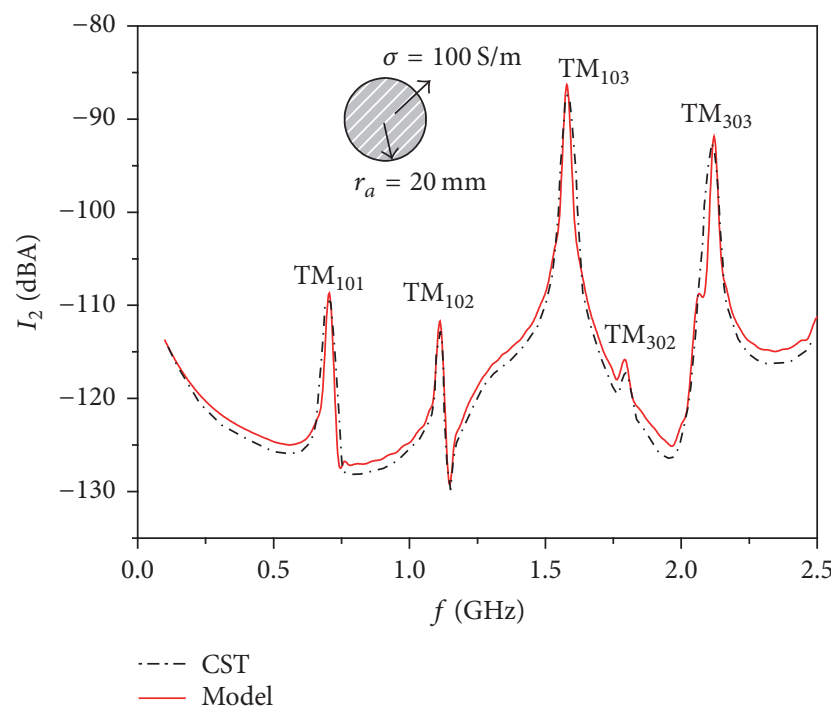

FIGURE 6: Electric current induced at termination load $Z_{2}$ for a covered circular aperture from the analytical model and the CST.

has different values; the resonance modes of them are different; for example, there are no resonances $\mathrm{TM}_{102}$ and $\mathrm{TM}_{302}$ for $z_{s}=-140 \mathrm{~mm}$, while $\mathrm{TM}_{102}$ and $\mathrm{TM}_{302}$ resonances exist, and there is a new resonance for $z_{s}=-170 \mathrm{~mm}$. It is because the coupling differs between the electric fields in enclosure 2 and the TL, and the electric dipole is at the position where the leakage electric field $E_{y}^{2}$ is zero for those missing resonance modes.

Figure 8 shows the electric current induced at termination load $Z_{1}$ for different conductivities of the sheet of $\sigma=20 \mathrm{~S} / \mathrm{m}$, $60 \mathrm{~S} / \mathrm{m}$, and $120 \mathrm{~S} / \mathrm{m}$. It can be seen that the larger the conductivity of the sheet is, the higher the induced current is. The reason is that the larger the conductivity of the sheet, 


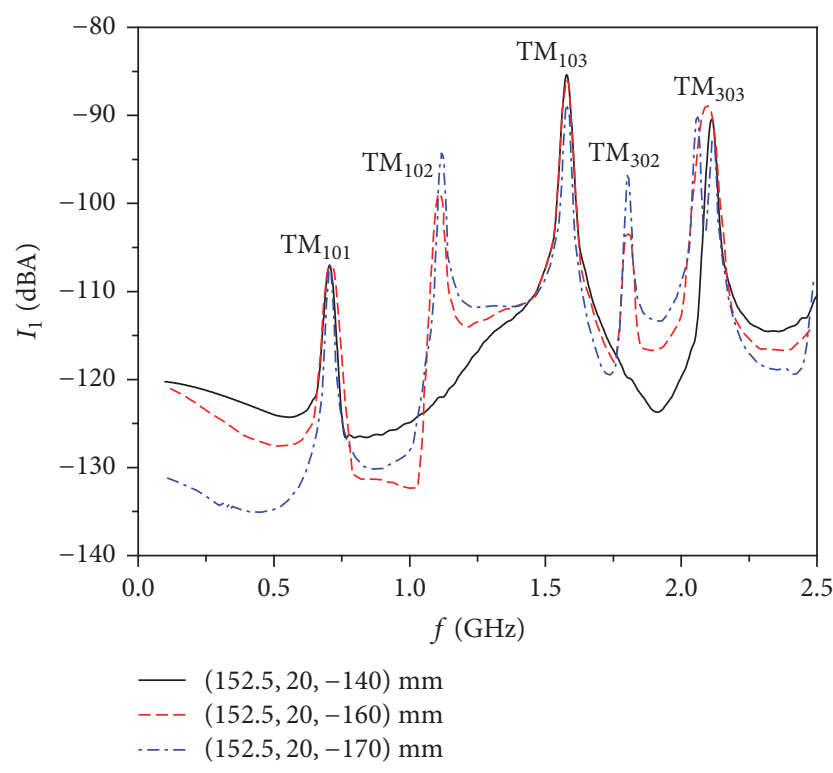

FIGURE 7: Electric current induced at termination load $Z_{1}$ for the electric dipole of different positions.

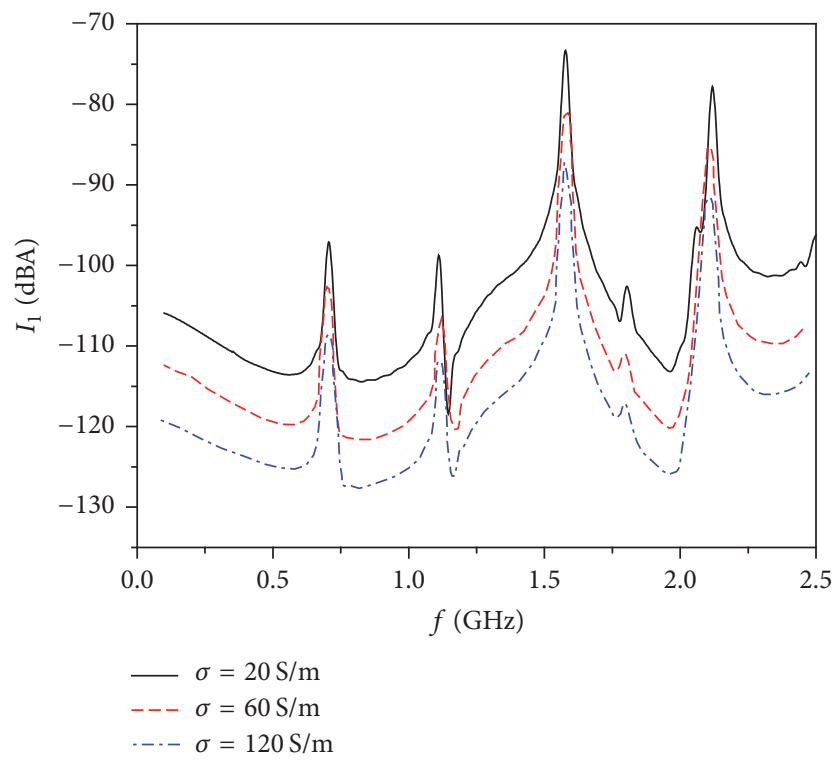

FIGURE 8: Electric current induced at the termination load $Z_{1}$ for different sheet conductivities.

the larger the corresponding equivalent magnetic current for the aperture, and thus the larger the electromagnetic field in enclosure 2, and the stronger the coupling between the electromagnetic field and the TL.

The proposed model is also suitable for multiple apertures by the superposition of the electromagnetic field generated by the equivalent magnetic current of each aperture. Figure 9(a) shows the case of an array of four circular apertures respectively covered by a conductive sheet of thickness $t=1 \mathrm{~mm}$ and conductivity $\sigma=100 \mathrm{~S} / \mathrm{m}$. In order to make the total area of the aperture array the same as that of the previous single circular covered aperture, the radius of each aperture is $r_{a}=$
$10 \mathrm{~mm}$. The separation distances along the $x$-axis and $y$-axis are $d_{h}=d_{v}=30 \mathrm{~mm}$ for neglecting the coupling between the apertures. Figure 9(b) shows the comparison of the electric current induced at the termination load $Z_{1}$ between the single aperture and multiple apertures of the same area. It can be seen that the induced current of the single aperture is higher than that of multiple apertures, indicating that the shielding effect of multiple apertures is better than that of the single aperture of the same area.

Keeping the location of the center point of the TL unvaried, Figure 10(a) shows the case of the TL oriented along the $z$-axis within the original enclosures. Figure 10 (b) is the corresponding side view of (a). Figure 10(c) shows the electric current induced at the termination loads $Z_{1}$ and $Z_{2}$ using our model.

By coordinate transformation, the proposed model is also appropriate for $x$-oriented or $z$-oriented electric dipole. Based on superposition principle, the model then can be expanded to deal with the electric dipole of arbitrary direction. Figure 11(a) shows an electric dipole with the elevation angle $\theta$ $=45^{\circ}$ and the azimuth angle $\varphi=30^{\circ}$. Figure 11(b) shows the electric current induced at the termination loads $Z_{1}$ and $Z_{2}$ corresponding to (a).

3.2. TL Outside the Enclosures. For the model in Figure 3, we assume that both of the two covered circular apertures are of radius $r_{a}=r_{b}=20 \mathrm{~mm}$, thickness $t_{1}=t_{2}=1 \mathrm{~mm}$, and conductivity $\sigma_{1}=\sigma_{2}=100 \mathrm{~S} / \mathrm{m}$. The center point of the TL and the observation point are, respectively, located at (150, 60, $450) \mathrm{mm}$ and $(150,60,450) \mathrm{mm}$ and other parameters of the multiple enclosures are equivalent to those in Section 3.1.

Figure 12 shows the comparison of $E_{y}^{\text {out }}$ using the analytical model and the results from the CST. Figure 13 shows the comparison of the electric current induced at the termination load $Z_{1}$ using the analytical model and the results from the CST. It can be seen that the two curves are in good agreement up to $2.5 \mathrm{GHz}$ except some frequency range, and all resonance modes (see Figures 12 and 13) have been identified.

For the case of the enclosures with apertures in multiple sides, by the superposition of the electromagnetic field generated by the equivalent magnetic current of each aperture, and then combining the equivalent $\mathrm{T}$ circuital model of the $\mathrm{TL}$ and the Agrawal coupling model, the electric current induced at the termination of the TL can be obtained. Based on Figure 3, Figure 14(a) shows enclosure 2 with another covered circular aperture of radius $r_{c}=20 \mathrm{~mm}$, thickness $t_{3}=1 \mathrm{~mm}$, and conductivity $\sigma_{3}=100 \mathrm{~S} / \mathrm{m}$, and the center point of the TL is located at $(0,60,450) \mathrm{mm}$. Figure $14(\mathrm{~b})$ shows the comparison of the electric current induced at the termination load $Z_{1}$ using the analytical model and the results from the CST. It can be seen that the two curves present good agreement in most frequency range up to $2.5 \mathrm{GHz}$.

\section{Conclusions}

In this paper, an efficient analytical method has been developed to predict the electromagnetic leakage field coupling with a lossless two-conductor TL located inside and outside 


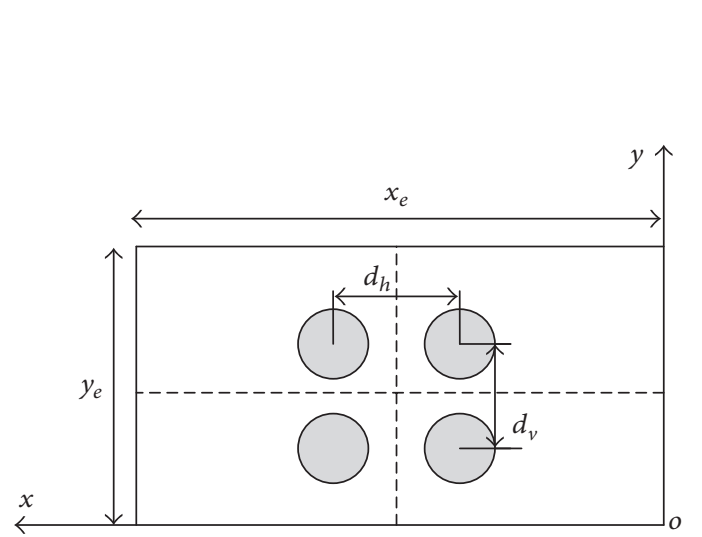

(a)

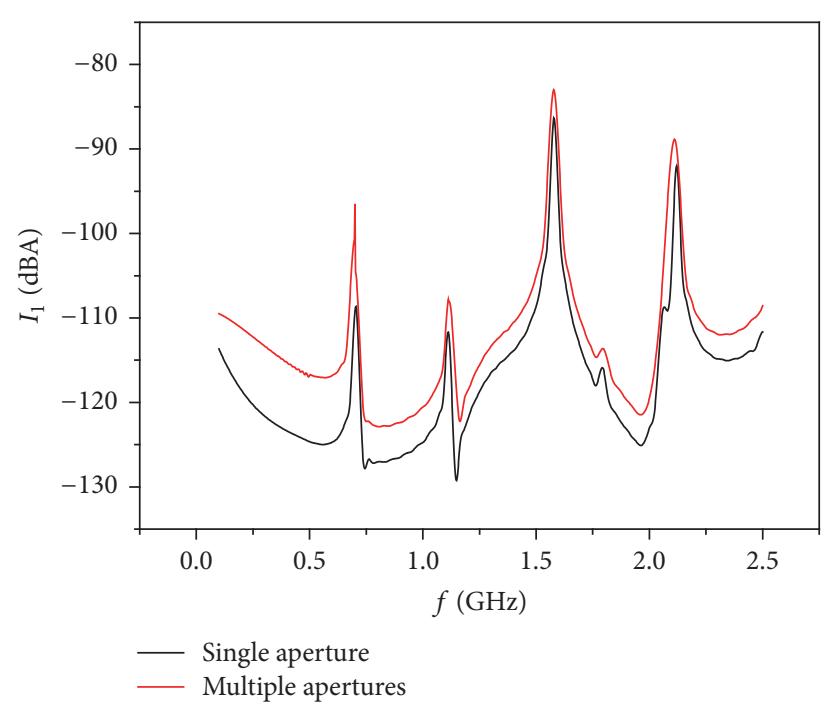

(b)

FIGURE 9: (a) An array of four circular apertures respectively covered by a conductive sheet of thickness $t=1 \mathrm{~mm}$ and conductivity $\sigma=$ $100 \mathrm{~S} / \mathrm{m}$. (b) Comparison of the electric current induced at termination load $Z_{1}$ between the single aperture and multiple apertures of the same area.

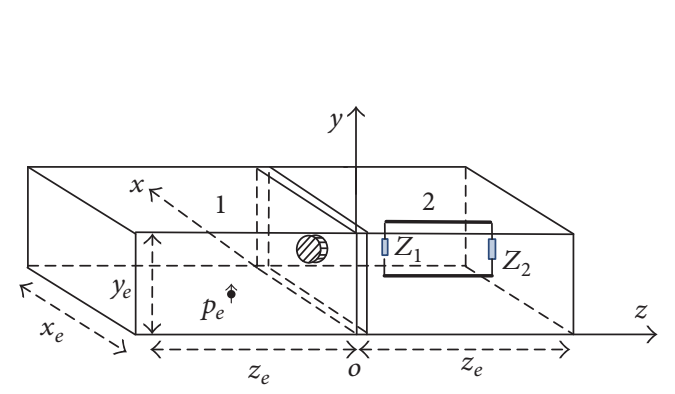

(a)

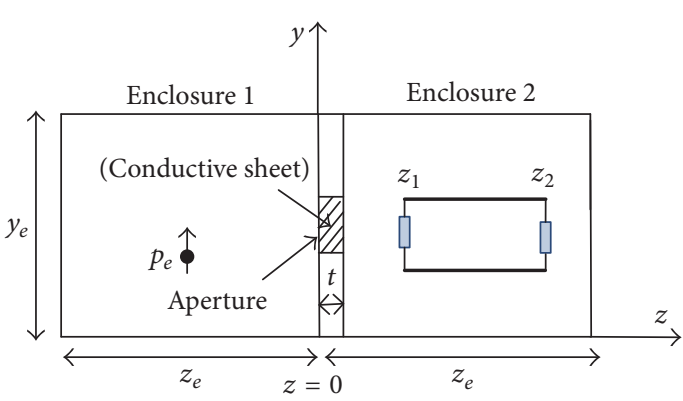

(b)

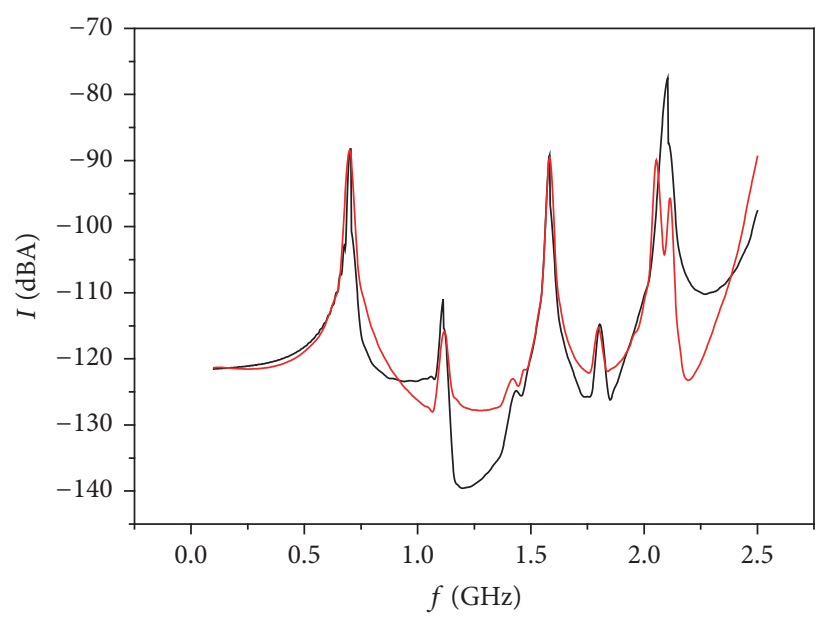

$$
\begin{array}{r}
-I_{1}\left(Z_{1}\right) \\
-I_{2}\left(Z_{2}\right)
\end{array}
$$

(c)

Figure 10: (a) The geometry of the enclosures with the TL oriented along the $z$-axis. (b) The side view of the enclosures corresponding to (a). (c) The electric current induced at the termination loads $Z_{1}$ and $Z_{2}$ for the TL oriented along the $z$-axis. 


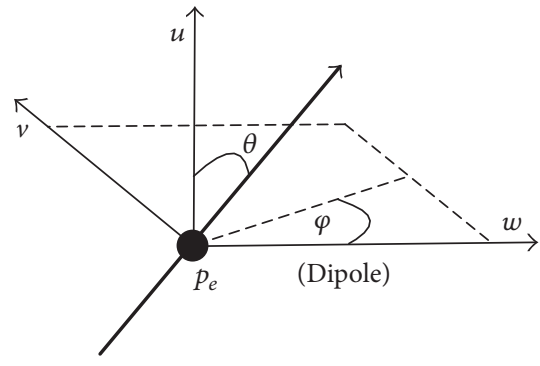

(a)

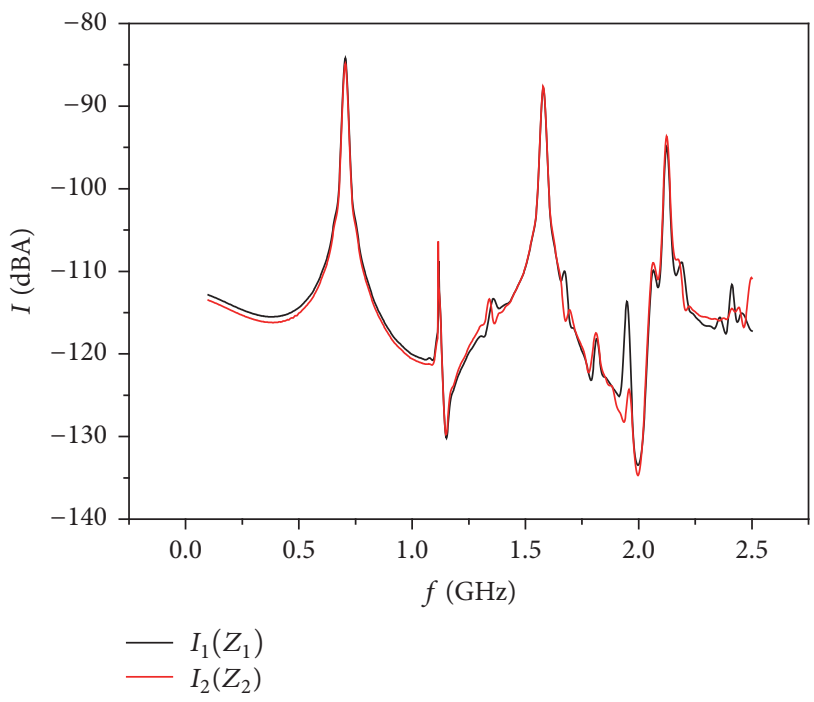

(b)

Figure 11: (a) An arbitrary directed electric dipole of the elevation angle $\theta=45^{\circ}$ and the azimuth angle $\varphi=30^{\circ}$. (b) The electric current induced at termination loads $Z_{1}$ and $Z_{2}$ for the arbitrary directed electric dipole.

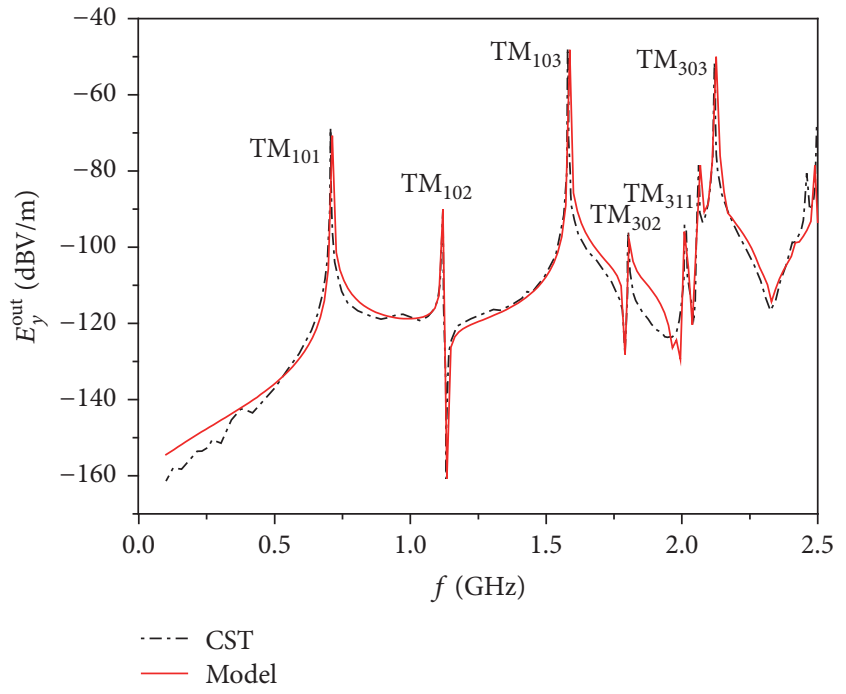

Figure 12: Component of electric field $E_{y}^{\text {out }}$ at $(150,60,450) \mathrm{mm}$ from the analytical model and the CST.

a rectangular enclosure through circular apertures covered by conductive sheets. We use the dyadic Green function and the boundary condition of the electromagnetic wave normally incident upon an infinite conducting surface in order to derive the leakage fields excited by the electric dipole within another adjacent rectangular enclosure. Then, we combine the equivalent lumped-T circuit model of the TL with the Agrawal coupling model to evaluate the electric current induced at the termination loads of the TL. The results from the analytical model are in good agreement with those from the full-wave simulation software CST in broad frequency range up to $2.5 \mathrm{GHz}$. Finally, the analytical

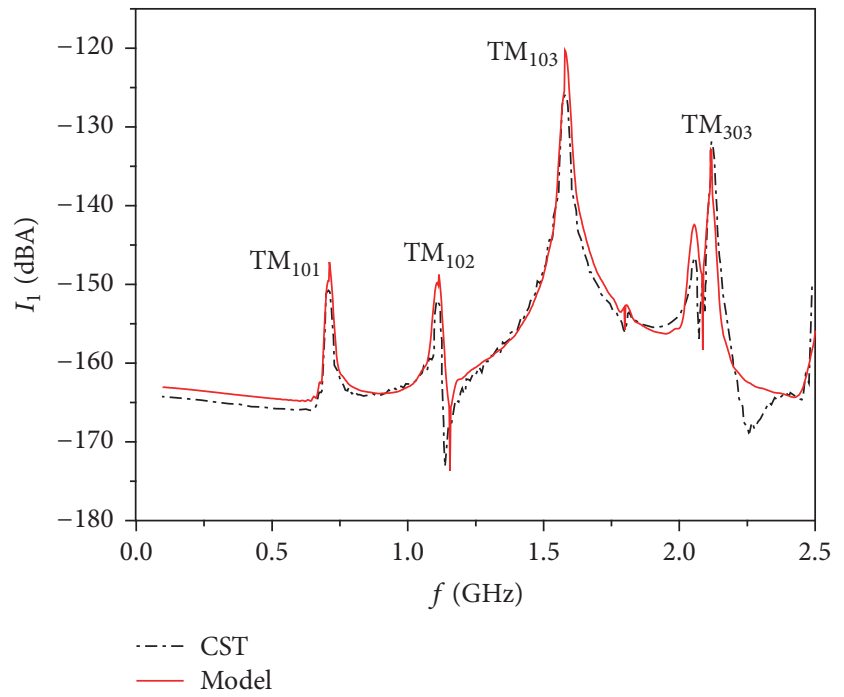

FIGURE 13: Electric current induced at termination load $Z_{1}$ from the analytical model and the CST.

model is employed to analyze the effect of different factors on the induced current, including the position of the electric dipole, the conductivity of the sheets, the quantity of the aperture, and the direction of the TL. The analytical model can also deal with apertures in multiple sides of the enclosures and electric dipole of arbitrary direction. The results show that the position of the electric dipole has a significant influence on both of the amplitude of the induced current and the resonance modes coupled with the TL; the larger the conductivity of the sheet is, the higher the induced current is; the shielding effect of multiple apertures is better than that of the single aperture of the same area. 


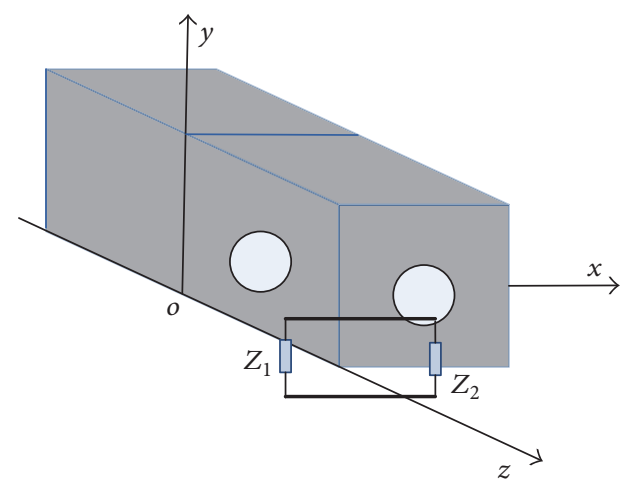

(a)

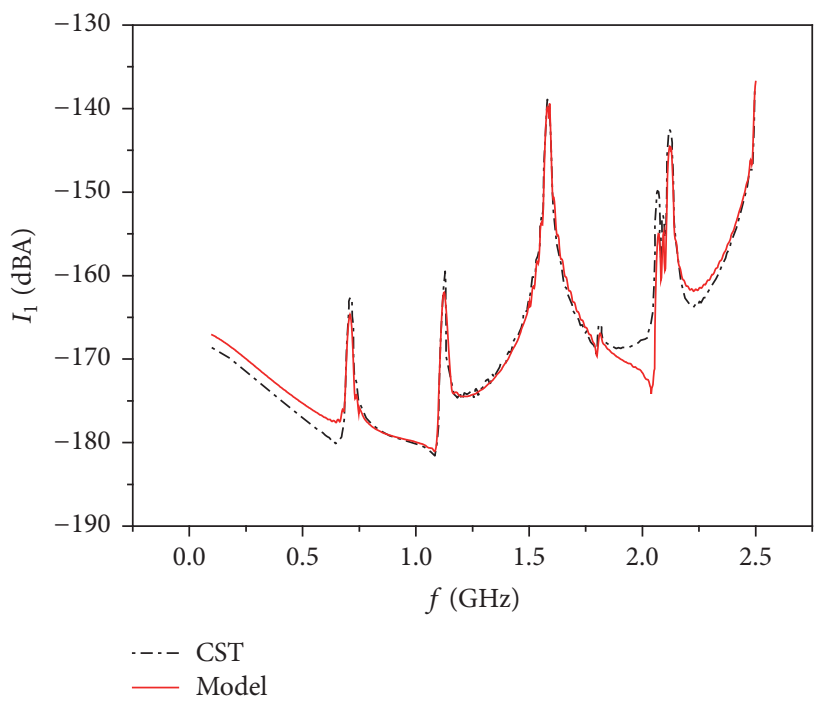

(b)

FIGURE 14: (a) The geometry of the enclosures with covered circular apertures in multiple sides. (b) Electric current induced at termination load $Z_{1}$ for apertures in multiple sides from the analytical model and the CST.

\section{Competing Interests}

The authors declare that there is no conflict of interests regarding the publication of this paper.

\section{Acknowledgments}

This work was supported by the National Natural Science Foundation of China (Grant no. 61372050) and the Fundamental Research Funds for the Central Universities in China (Grant no. 2016MS06).

\section{References}

[1] T. Yang, Coupling onto radio frequency components enclosed within canonical structures [Ph.D. thesis], University of Michigan, Ann Arbor, Mic, USA, 2006.

[2] S. Abadpour, P. Dehkhoda, R. Moini, and S. H. H. Sadeghi, "Susceptibility of a shielded and an unshielded microstrip transmission line against a near interference source," Universal Journal of Electrical and Electronic Engineering, vol. 2, no. 3, pp. 137-143, 2014.

[3] S. Tkachenko, J. Nitsch, and R. Rambousky, "Electromagnetic filed coupling to transmission lines inside rectangular resonators," Interaction Notes 623, 2011.

[4] H. Xie, J. Wang, D. Sun, R. Fan, and Y. Liu, "Analysis of EMP coupling to a device from a wire penetrating a cavity aperture using transient electromagnetic topology," Journal of Electromagnetic Waves and Applications, vol. 23, no. 17-18, pp. 2313-2322, 2009.

[5] J. P. Parmantier, "First realistic simulation of effects of EM coupling in commercial aircraft wiring," Computing \& Control Engineering Journal, vol. 9, no. 2, pp. 52-56, 1998.

[6] Y. Li, J. Luo, G. Ni, and J. Shi, "Electromagnetic topology analysis to coupling wires enclosed in cavities with apertures,"
Mathematical Problems in Engineering, vol. 2010, Article ID 209591, 11 pages, 2010.

[7] A. Boutar, A. Reineix, C. Guiffaut, and G. Andrieu, "An efficient analytical method for electromagnetic field to transmission line coupling into a rectangular enclosure excited by an internal source," IEEE Transactions on Electromagnetic Compatibility, vol. 57, no. 3, pp. 565-573, 2015.

[8] T. Konefal, J. F. Dawson, A. C. Marvin, M. P. Robinson, and S. J. Porter, "A fast multiple mode intermediate level circuit model for the prediction of shielding effectiveness of a rectangular box containing a rectangular aperture," IEEE Transactions on Electromagnetic Compatibility, vol. 47, no. 4, pp. 678-691, 2005.

[9] T. Konefal, J. F. Dawson, A. C. Marvin, M. P. Robinson, and S. J. Porter, "A fast circuit model description of the shielding effectiveness of a box with imperfect gaskets or apertures covered by thin resistive sheet coatings," IEEE Transactions on Electromagnetic Compatibility, vol. 48, no. 1, pp. 134-144, 2006.

[10] T. Konefal, J. F. Dawson, A. C. Denton et al., "Electromagnetic coupling between wires inside a rectangular cavity using multiple-mode-analogous-transmission-line circuit theory," IEEE Transactions on Electromagnetic Compatibility, vol. 43, no. 3, pp. 273-281, 2001.

[11] A. Nanni, D. W. P. Thomas, C. Christopoulos et al., "Electromagnetic coupling between wires and loops inside a rectangular cavity using multiple mode transmission line theory," in Proceedings of the International Symposium on Electromagnetic Compatibility (EMC Europe '04), vol. 2, pp. 609-614, Eindhoven, The Netherlands, September 2004.

[12] M.-D. Chen, X.-H. Jie, and H.-Y. Zhang, "Simulation and calculation of the absorbing microwave properties of carbon nanotube composite coating," Wuli Xuebao/Acta Physica Sinica, vol. 63, no. 6, pp. 188-193, 2014 (Chinese).

[13] G. Andrieu, J. Panh, A. Reineix et al., "Homogenization of composite panels from a near-field magnetic shielding effectiveness measurement," IEEE Transactions on Electromagnetic Compatibility, vol. 54, no. 3, pp. 700-703, 2012. 
[14] F. M. Tesche, M. V. Ianoz, and T. Karlsson, EMC Analysis Methods and Computational Models, John Wiley \& Sons, New York, NY, USA, 1997.

[15] C. Q. Jiao and Y. Y. Li, "Reciprocity principle-based model for shielding effectiveness prediction of a rectangular cavity with a covered aperture," Chinese Physics B, vol. 24, no. 10, Article ID 104101, pp. 1-6, 2015.

[16] M. D. Deshpande, "Electromagnetic field penetration studies," NASA Technical Paper, 2000.

[17] C. R. Paul, Analysis of Multiconductor Transmission Lines, John Wiley \& Sons, New York, NY, USA, 1993. 

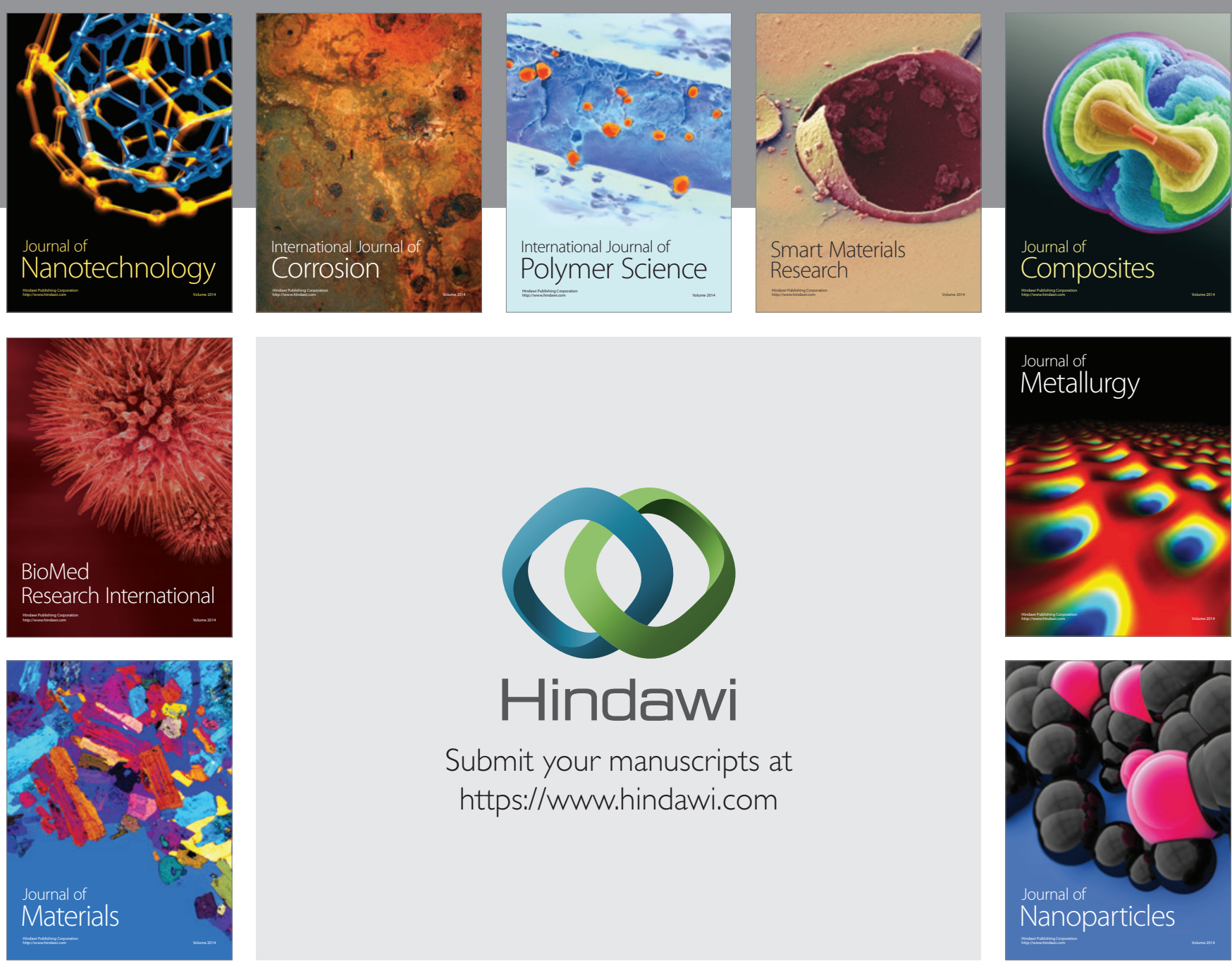

\section{Hindawi}

Submit your manuscripts at

https://www.hindawi.com

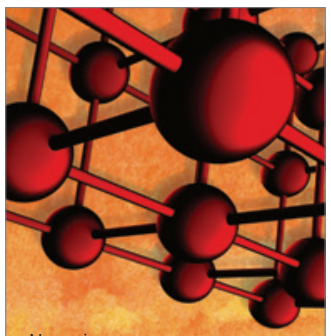

Materials Science and Engineering
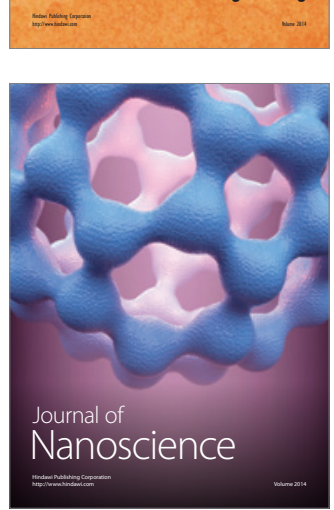
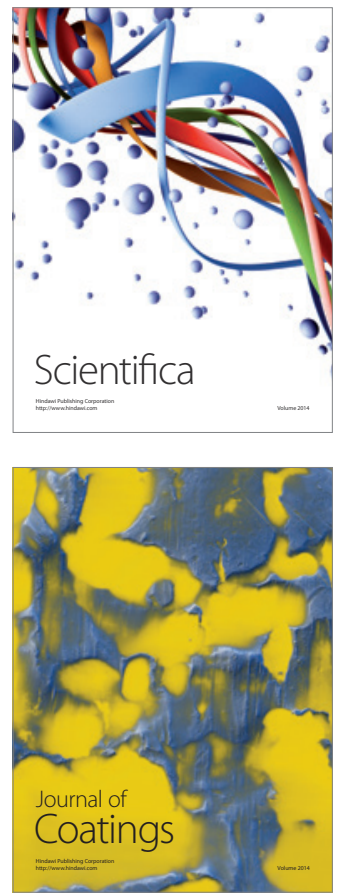
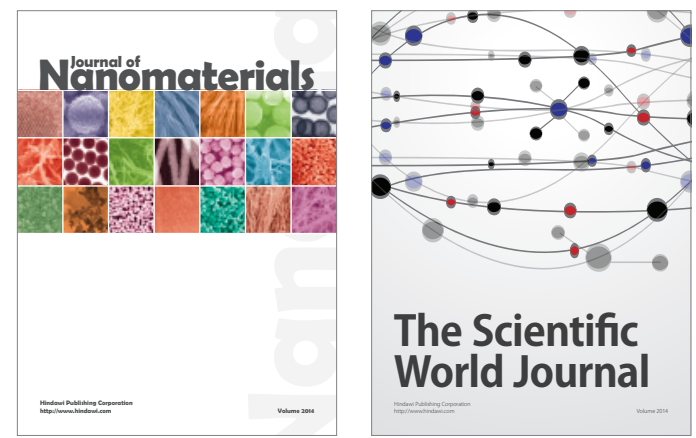

The Scientific World Journal
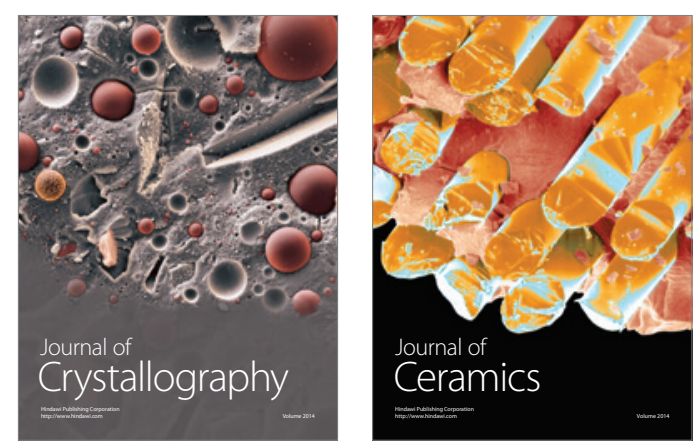
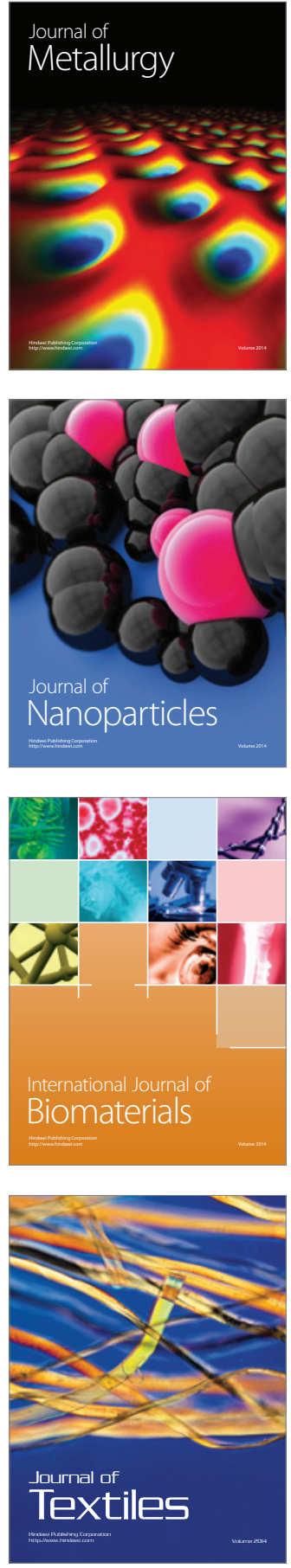